\title{
Basics of Biopharmaceutics: A Mini Review
}

\author{
Lakshmana Prabu S1* and Suriyaprakash TNK ${ }^{2}$ \\ ${ }^{1}$ Department of Pharmaceutical Technology, Bharathidasan Institute of Technology, \\ Anna University, Tiruchirappalli, India \\ 2Department of Pharmaceutics, Al Shifa College of Pharmacy, Kerala, India
}

\section{Mini Review \\ Volume 1 Issue 1}

Received Date: May 10, 2017

Published Date: June 29, 2017

\section{*Corresponding author: Lakshmana Prabu S, Department of Pharmaceutical}

Technology, Bharathidasan Institute of Technology, Anna University, Tiruchirappalli, India, Tel: +919750550965; Email: slaxmanvel@gmail.com

\section{Abstract}

Drug research is a unique multi-disciplinary process towards the development of novel therapeutic agent to meet the current global medicinal needs. Drug discovery and development are the two major stages in drug research. Drug discovery includes new target identification and characterization, synthesis of new lead molecules, physicochemical characterization and screening of the lead molecule for its biological activity through in vitro and in vivo techniques. Drug development stage includes the assessment of its safety and toxicity of the developed new drug molecule. Drug molecules are substance used for diagnosis or treating for various diseases. Drugs can be given in different dosage form such as solids, semisolids, liquids, topical and parenteral preparation to produce the desired therapeutic activity and to meet the patient's needs. Biopharmaceutics is a major branch in pharmaceutical sciences describes the role of dosage form in the absorption and disposition of drugs in the body which are assessed through pharmacodynamic and pharmacokinetic parameters or properties. Pharmacodynamics correlates the concentration of the drug with its therapeutic and toxicological effect. Pharmacokinetics correlates how the drug moves through the human body by its four main pathways such as absorption, distribution, metabolism and elimination and measuring the drug concentration in biological matrices.

Keywords: Pharmacokinetics; in vitro and in vivo; Pharmacodynamics; Bioavailability; Endocytosis

\section{Introduction}

Drug discovery and development are the two major stages in drug research to meet the current global medicinal needs. Target identification and its characterization, synthesis of new chemical entity and its characterization, evaluation of biological activity through in vitro and in vivo techniques are involved in drug discovery. To develop a new chemical entity around 10-14 years are needed with an average cost of more than $\$ 800$ million. Success rate of the drug discovery and development is approximate of $0.01 \%$ [ 1 out of 10,000 screened compounds] [1,2]. Human body is composed as series of membrane barriers which are divided into compartment filled with aqueous components. These membrane barriers are composed of phospholipid bilayers; these phospholipid membranes consist of head group (polar) and tail groups (non-polar). Drugs lipophilicity and partition coefficient are important for membrane permeability $[3,4]$. 


\section{Bioequivalence \& Bioavailability International Journal}

\section{Biopharmaceutics}

Biopharmaceutics is a major branch in pharmaceutical sciences which has an important role in drug discovery and development to ensure the therapeutic efficiency of the developed drug molecule. Biopharmaceutics links pharmacodynamic and pharmacokinetic properties of the drug substance. In short, biopharmaceutics mainly involves drug absorption process. The other parameters like aqueous solubility, stability, permeability and first pass effect are also concern in biopharmaceutics [5,6]. Knowledge of biopharmaceutics is crucial to adjust the drug dosing among the different species and also the role of drug does to the body and what body does to the drug $[7,8]$.

\section{Pharmacodynamics}

Pharmacodynamics correlates the concentration of the drug with its therapeutic and toxicological effect.

\section{Pharmacokinetics}

Pharmacokinetics deals with the measurement of drug concentration in biological matrices by selective and sensitive bioanalytical methods based on the drug's absorption, distribution, metabolism and excretion (ADME). Understanding of pharmacokinetic properties is important in drug discovery and development process; poor pharmacokinetic properties of any drug has exclusive role in termination from further studies $[1,9,10]$.

\section{Pharmacokinetic parameters}

Absorption: Concentration of drug in the blood and other biological matrix after drug administration reflects the absorption of the drug. Absorption of the drug also reflects the solubility as well as the crystal nature of the drug.

Various factors which affect the absorption of the drug are:

1. Physicochemical characteristics of the drug

2. Formulation factors

3. Physiological factors affecting bioavailability $[6,11]$.

Absorption of Drug: Drugs are either weak acid or base in nature; it exist both ionized and non-ionized forms in the body. In membrane lipids, non-ionized form of drug is sufficiently soluble and it can cross the biological cell membrane. The protonated form is predominates when the $\mathrm{pH}$ is less than $\mathrm{pKa}$, whereas non-protonated form is predominates when the $\mathrm{pH}$ is greater than $\mathrm{pKa}$. When weak acid drugs are protonated it becomes non-ionized form and readily absorb in stomach, when weak basic drugs are protonated it becomes ionized and readily absorb in the intestine.
Drugs can be absorbed after crossing the biological membrane. Drugs can cross the biological membrane either transcellular or paracellular mechanism $[5,12]$.

\section{Drug transport across cell membranes:}

1. Passive diffusion: In Passive diffusion, the drug transport across the cell membrane based on the concentration gradient which provides energy. In passive diffusion process drug in the form of unionized form is absorbed in higher concentration than ionized form.

2. Active transport: In active transport, drug molecules move across the cell membrane from the lower concentration to higher concentration by utilizing the chemical energy.

3. Endocytosis: In endocytosis, molecules are absorbed by engulfing by utilizing energy.

The other endocytosis type processes are exocytosis, phagocytosis and pinocytosis [11,13-14].

Evaluation techniques of drug absorption: The rate and extent of drug absorption can be evaluated through various in vitro and in vivo techniques.

Human colon adenocarcinoma cell lines Caco-2, HT29 and MDCK are commonly utilized to assess the drug absorption. Other in vitro models utilized to assess the drug absorption are cell culture models, isolated mucosal cells, brush border membrane vesicles, isolated tissue techniques and everted tissue techniques includes everted sac technique and intestinal rings [1518].

\section{Distribution}

Distribution gives information about the time duration of drug in tissue or organs. In simple way distribution is a reversible transfer of drug within the body from one location to another. Lipophilicity and protein binding are the two factors having an important role drug distribution in the body. Lipophilic drugs will get accumulated in organs and tissues whereas hydrophilic drugs will get accumulated in cells.

Distribution of the drug is depends upon the following factors, such as:

$>$ Blood flow to the tissue

$>$ Ratio between tissue/blood

$>$ Apparent Volume of Distribution

$>$ Protein binding

$>$ Lipid solubility

$>$ Molecular Size

$>$ Ionization of drug

$>$ Permeation across the cell membrane Compartment models in drug distribution 
After administration, distribution of drug in a biological system can be explained through hypothetical structures as compartment models.

Various pharmacokinetic compartment models in drug distribution are:

$>$ One-compartment model

$>$ Two-compartment model

$>$ Multi-compartment model [19-21].

\section{Drug Metabolism}

Drug metabolism is conversion of drug into its metabolites either active or inactive through enzymes. In simple way drug metabolism is a process to facilitate the excretion of drug by converting the drug into less polar or more polar and water soluble. Most of the drugs are metabolized in the liver, which converts the lipophilic drugs into hydrophilic drugs through metabolizing enzymes. Other organs involved in the metabolism of drugs through metabolizing enzymes are gut, kidneys, brain, lungs and skin.

Usually drug metabolism is carried out by two processes such as:

1. Phase I- Unmasking functional group like $\mathrm{OH}, \mathrm{NH}_{2}$, SH which facilitate the phase II conjugative reaction.

2. Phase II- Conjugation reaction with amino acid, acetic acid, sulfuric acid and glucuronic acid and convert the drug into more water soluble and readily excreted through urine or bile.

Classification of drug metabolizing enzymes:

1. Microsomal (inducible) - Mixed-function oxidases (Cytochrome P-450 reductase and NADPH), Glucuronyl transferase and enzymes involved in hydrolysis and oxidation.

2. Non-microsomal (non-inducible) - All conjugations except glucuronidation.

Non-microsomal enzymes metabolize the drugs through conjugation, oxidation, reduction and hydrolysis [22-24].

Evaluation techniques of drug metabolism:

1. In vitro methods - Assess the biochemical toxicology, cytotoxicity, irreversible drug protein binding, drug metabolism and enzyme regulation.

i. Enzyme system - Enzymes such as cytochrome P450, CYP1A, CYP2C, CYP2D, CYP2E, CYP3A and CYP4A.

ii. Subcellular fractions - Microsomes as subcellular fraction composed of endoplasmic reticulum and enzymes such as flavin monomoxygenases, glucuronyltransferase, epoxide hydrolases, alcohol dehydrogenases, esterase and methyltransferases.

iii. Cellular systems - Cellular system is used to assess the drug metabolism, permeation of drug across the cell membrane, enzyme stimulation and cytotoxicity.

iv. Organ slices - Various organ slices such as liver, brain, heart and kidney are used to assess its biochemical process.

2. In vivo method

i. Radionuclides - Radionuclides such as Tritium $\left({ }^{3} \mathrm{H}\right)$ and Carbon14 $\left({ }^{14} \mathrm{C}\right)$ are used to assess the drug metabolism $[25,26]$.

\section{Clearance}

Drug clearance (CL) is defined as the quantity of drug cleared per unit time from the vascular compartment through metabolism and excretion. In general drugs are extracted from the body through hepatic clearance and renal excretion.

\section{Excretory organs}

Major routes: Kidneys, Liver and Lungs

Minor routes: Skin, hair, sweat, saliva, tears and milk [6,11,27-30].

\section{Conclusion}

Drug research is a unique multi-disciplinary process towards the development of new chemical entity to meet the current medical need of various diseases. Various new techniques such as combinatorial chemistry, molecular modeling and high throughput screening are used to choose a potential drug for discovery and development. Majority of the drug molecules fails during the drug development process due to its biopharmaceutical properties. The basic concept of pharmacokinetic is quantification of clearance rate, biological half-life, extent of protein binding and volume of distribution in the biological matrix of the drug and its metabolites by selective and sensitive bioanalytical method. Preliminary screening of pharmacokinetic properties is essential and have important role in the drug discovery and development. During the drug development any drug has poor pharmacokinetic properties it will be excluded from the further process to fulfill the current medicinal needs.

\section{Reference}

1. Lakshmana Prabu S, Thirumurugan R, Suriyaprakash TNK, Ruckmani K (2014) The Role of the Drug Discovery, Clinical, and Regulatory Affairs Teams in Turning a Potent Agent into a Registered Product. In: Elsevier Reference Module in 


\section{Bioequivalence \& Bioavailability International Journal}

Chemistry, Molecular Sciences and Chemical Engineering, Reedijk J. (Ed.) Elsevier, Waltham, MA.

2. Pharmaceutical Research and Manufacturers of America (PhRMA), Pharma FAX of the Week, May 2, 2003, R\&D Process: Long, Expensive, Risky Road.

3. Pliska V, Testa B, Van de Waterbeemd H (1996) Lipophilicity in Drug Action and Toxicology. VCH, Weinheim J Med Chem 39(26): 5287-5288.

4. Smith DA, Allerton C, Kalgutkar, Waterbeemed H, Walker DK, et al. (2012) In: Pharmacokinetics and Metabolism in Drug Design. Phytochemistry 51.

5. Mehrotra N, Gupta M, Kovar A, Meibohm B (2007) the role of pharmacokinetics and pharmacodynamics in phosphodiesterase-5 inhibitor therapy. Int J Impot Res 19: 253-264.

6. Panchagnula R, Thomas NS (2000) Biopharmaceutics and pharmacokinetics in drug research. Int J Pharmaceutics 201: 131-150.

7. Lin JH, Lu AY (1997) Role of Pharmacokinetics and Metabolism in Drug Discovery and Development. Pharmacol Rev 49(4): 403-449.

8. Gunaratna C (2000) Drug metabolism and pharmacokinetics in drug discovery: a primer for bioanalytical chemists, part I. Current separation 19: 17-23.

9. Lakshmana Prabu S, Suriya Prakash TNK (2012) Extraction of Drug from the biological matrix: A review. In: Applied biological engineering principles and practice. Naik GR (Eds.), InTech, Croatia, 479-506.

10. Ali AS, Abdel-Rahman MS, Rahman F, Osman $\mathrm{OH}$ (2013) Basic Principles of Therapeutic Drug Monitoring. J Appl Biopharm Pharmacokinet 1: 8795.

11. Satoskar RS, Bhandarkar SD, Nimala NR (1993) In: Pharmacology and Pharmacotherapeutics. (ed.), Popular Prakashan Private Ltd, Mumbai, pp: 1-50.

12. http://www.us.elsevierhealth.com/media/us/samp lechapters/9781416066279/Chapter \%2002.pdf [Accessed on 10.05.2017].

13. Levine RR. (2000) Pharmacology: Drug Actions and Reactions, $6^{\text {th }}$ (edn.) New York, Parthenon.

14. Brahmankar DM, Jaiswal SB, Vallabh prakashan (1995) In: Biopharmaceutics and pharmacokinetics a treatise. Absorption of drugs. New Delhi, pp: 5-75.
15. Nicolazzo JA, Finnin BC (2008) In vivo and in vitro models for assessing drug absorption across the buccal mucosa. Drug Absorption Studies 7: 89-111.

16. Hubatsch I, Ragnarsson EGE, Artursson P (2007) Determination of drug permeability and prediction of drug absorption in Caco-2 monolayers. Nat Protocols 2(9): 2111-2119.

17. Quan Y, Jin Y, Faria TN, Tilford CA, He A, et al. (2012) Expression Profile of Drug and Nutrient Absorption Related Genes in Madin-Darby Canine Kidney (MDCK) Cells Grown under Differentiation Conditions. Pharmaceutics 4(2): 314-333.

18. Habucky K (2005) Methods to assess absorption in drug discovery, In: Pharmacokinetics RegulatoryIndustrial-Academic Perspective. Welling PG and Tse FLS (Ed.), Marcel Dekker, NY67: 21-37.

19. Smith DA, van de Waterbeemd $\mathrm{H}$, Walker DK, Mannhold R, Kubinyi H, et al. (2001) In: Pharmacokinetics and Metabolism in Drug Design., Timmerman H (Ed.), Wiley-VCH Verlag GmbH, Weinheim 51: 47-57.

20. Kari A, Sateesh G, Suresh B, Rao BSS (2012) Factors Effecting on Drug Distribution. The pharma innovation 1(6): 69-73.

21. Blomhoj M, Kjeldsen TH, Ottesen J (2014) Compartment models. Pp: 1-47.

22. Smith DA, van de Waterbeemd $H$, Walker DK, Mannhold R, Kubinyi H, et al.(2001). In: Pharmacokinetics and Metabolism in Drug Design. (Ed.), Biotransformation of drugs. Wiley-VCH Verlag GmbH, Weinheim, pp. 75-98.

23. Handschin C, Meyer UA (2003) Induction of drug metabolism: the role of nuclear factors. Pharmacol Rev 55(4): 649-673.

24. Benet LZ, Oie S, Schwartz JB (1995). In: Goodman and Gillman's The Pharmacological Basis for Therapeutics, McGraw-Hill, New York, pp: 17071792.

25. Hayes RH, Pool WF, Sinz MW, Woolf TF (2005) Recent developments in drug metabolism methodology. In: Pharmacokinetics RegulatoryIndustrial-Academic Perspective. Welling PG, Tse FLS, Marcel Dekker, NY, 67: 201-234.

26. Lakshmana Prabu S, Suriya Prakash TNK, Ruckmani K, Thirumurugan R. (2015) Biopharmaceutics and pharmacokinetics. In: Basic Pharmacokinetic Concepts and Some Clinical Applications. Ahmed TA. 


\section{Bioequivalence \& Bioavailability International Journal}

27. Smith DA, van de Waterbeemd $H$, Walker DK, Mannhold R, Kubinyi $H$ et al. (2001) In: Pharmacokinetics and Metabolism in Drug Design., pp. 59-66.

28. Smith DA, van de Waterbeemd $H$, Walker DK, Mannhold R, Kubinyi H, (2001) In: Pharmacokinetics and Metabolism in Drug Design., Weinheim, enal clearance. pp. 67-74.
29. Brown D, Tomlin M (2010) Pharmacokinetic Principles. In: Pharmacology and pharmacokinetics A Basic reader. Tomlin M (ed.) Springer-Verlag London Limited, pp. 13-52.

30. Tracy TS. Metabolism and excretion of drugs. pp. 34-47. 